Article

\title{
Terahertz Wideband Filter Based on Sub-Wavelength Binary Simple Periodic Structure
}

\author{
Pan Yao ${ }^{1,2, \dagger}$, Huang H.X. ${ }^{1,3, \dagger}$, Lei Lei ${ }^{1,2}$, Zou Yang ${ }^{1,2}$, Xiao Y.F. ${ }^{1,2}$, Yang Tuo ${ }^{1,2}$ and Xu Ping ${ }^{1, *}$ \\ 1 Institute of Micro-Nano Optoelectronic Technology of Shenzhen University, Shenzhen 518060, China; \\ panyao2016@email.szu.edu.cn (P.Y.); fishon@163.com (H.H.X.); leilei@szu.edu.cn (L.L.); \\ zouyang@sredtech.com (Z.Y.); xallen@163.com (X.Y.F.); yangtuo@szu.edu.cn (Y.T.) \\ 2 College of Electronic Science and Technology, Shenzhen University, Shenzhen 518060, China \\ 3 College of Big Data and Internet, Shenzhen University of Technology (Finance), Shenzhen 518000, China \\ * Correspondence: xuping@szu.edu.cn; Tel.:+86-075526536099 \\ + These authors contributed equally to this work.
}

Received: 10 December 2018; Accepted: 17 January 2019; Published: 25 January 2019

\begin{abstract}
In this paper, a silicon-based terahertz wideband filter was designed using rigorous couple wave theory to achieve high diffraction efficiency and wideband filtering performance. The optimal parameters, such as filter period, duty ratio, and groove depth, are given. The design value and tolerance analysis were carried out to obtain the allowable deviation range of each structural parameter of the filter. The device was fabricated and tested for performance. The experimental results were consistent with the design results, proving the feasibility of the design method and can be applied in terahertz, communication, imaging, and other fields.
\end{abstract}

Keywords: terahertz; sub-wavelength; binary simple periodic structure; rigorous couple wave theory; genetic algorithm; wideband filter

\section{Introduction}

As an important part of terahertz functional devices, terahertz broadband filters have important research significance in terahertz technology applications such as medical imaging, security inspection, and product inspection. At present, the main categories of terahertz broadband filters are mainly focused on photonic crystal filters [1], plasma filters [2], and meta-material filters [3-9]. Photonic crystal filters mainly have terahertz broadband filters with low center-bands and narrow bands with multiple peaks. The filters can achieve a high transmittance of more than $90 \%$, but only achieve a narrow 3-dB bandwidth of several tens of GHz. Meanwhile, plasma filters use a new metal microstructure to design a low-free-wideband filter and can achieve a high transmittance of more than $80 \%$, but the 3-dB bandwidth is narrow, only reaching about $400 \mathrm{GHz}$. There are few reports on the design of structures of plasma filters. On the other hand, the meta-material terahertz filter has become the most popular in research due to its diversity of materials and broadband filters with excellent performance, high working frequency, and broad 3-dB bandwidth. However, the filter is difficult to manufacture due to its complicated structure, and therefore these structures are rarely fabricated.

In 2000, I. H. Libon et al. [3] illuminated the hybrid quantum well structure by laser, thereby achieving the combination of holes and electrons, as well as generating photons. This in turn changed the number of carriers in the structure. In 2011, Singapore National University et al. [4] used a flexible substrate to achieve a broadband filtering effect using polyethylene naphthalate (PEN) as a substrate, overlapping the multi-layer loop structure and fabricating a broadband filter. Among the abovementioned filters, Xuetong Zhou et al. [6] designed an ultra-wideband terahertz band-pass filter with a double-cone filter (DCF) with a 3-dB bandwidth of $2.2 \mathrm{THz}$ and a center frequency of about 
4.07 THz. The filter's highest transmittance was nearly $37 \%$. It is currently the filter with the widest 3-dB bandwidth and the highest center frequency.

The methods and structures reported above are relatively complicated, such as T-type and Y-type metal multilayer film structures, and fabrication and processing are difficult. The selected materials (e.g., gold, silver, and lithium niobate) are costly. Additionally, the filter has low transmission efficiency-nearly $37 \%$ in a terahertz band above $3 \mathrm{THz}$. Therefore, it is important to realize a broadband transmission filter with high transmission efficiency, low cost, and a simple structure.

Based on the research on sub-wavelength optical design and application accumulated by our research group [10-19], this paper proposes a method based on a simple periodic binary sub-wavelength structure $[12,13]$ to design a terahertz broadband filter. A silicon-based terahertz wideband filter with a center frequency of $4.07 \mathrm{THz}$ was designed. The theoretical 3-dB bandwidth is $2.33 \mathrm{THz}$, the highest transmission efficiency is nearly $50 \%$, and the bandwidth is $0.13 \mathrm{THz}$ wider than the best existing DCF. The maximum transmission efficiency is increased by more than ten percent. The device was further fabricated and tested, and the experimental results were consistent with the design results, which proved the feasibility of the design method. In this paper, high-performance broadband filtering was realized with a simple structure, which breaks through the limitations of current design and is convenient for mass production. It has important application value in terahertz communications and imaging.

\section{Design Principles and Results}

Figure 1 shows a binary sub-wavelength simple periodic structure whose refractive index is uniformly distributed in the $y$ direction, periodically distributed in the $x$ direction. $d$ is the period of the grating, and $a$ is the ridge width of the grating. In addition, $b$ is the groove width of the grating, $h$ is the groove depth of the grating, and $f=a / d$ is the duty ratio of the grating - that is, the ratio of the minimum line width of the grating to the grating period. In the $z$ direction, the structural region is divided into three regions - an incident region, a grating region, and a base region —within which $n_{1}$ is the refractive index of the incident region, where $z<0 ; n_{2}$ is the refractive index of the grating region as a grating layer, where $0<z<h ; n_{3}$ is the refractive index of the base region, where $z>h$; and the incident angle of the terahertz wave is normally incident (i.e., $\theta=0$ ). The thickness of the incident and base regions is considered to be infinite.

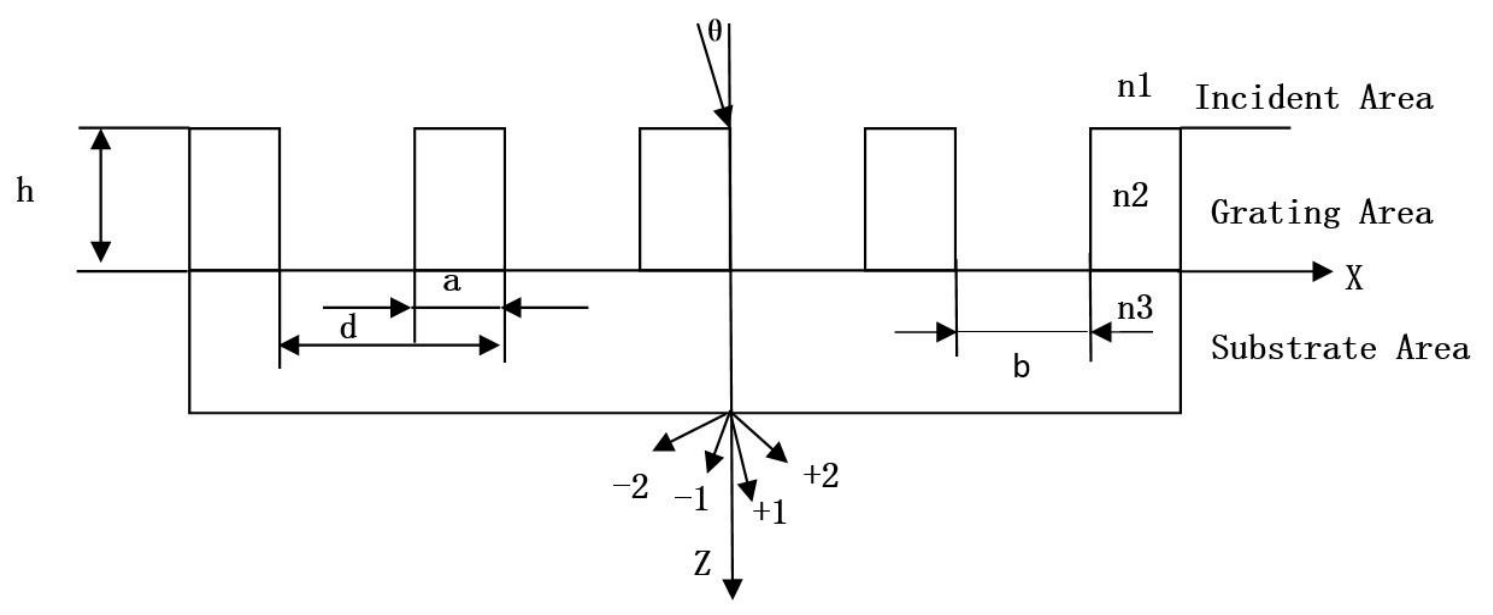

Figure 1. Sub-wavelength binary simple periodic structure model.

By utilizing the characteristics of the sub-wavelength structure, the diffraction order intensity in each of the diffracted waves generated by the incident wave passing through the grating can be adjusted by adjusting the grating structure parameters, such as the duty ratio $f$, the grating period $d$, and the groove depth $h$. 
By inhibiting the intensity of the non-zero diffraction order, an evanescent wave on the grating surface is excited to increase the intensity (i.e., transmission efficiency) of the zero-order diffraction. However, due to the strong coupling between the sub-wavelength structure and the incident wave, there is no analytical relationship between each structural parameter and the diffraction efficiency of each level, and the diffraction efficiency of each level is very sensitive to the change of structural parameters. That is, a slight structural change would cause a sharp change in the filtering performance. It can be seen that it is very difficult to obtain a broadband filter with high transmission efficiency by manually adjusting the structural parameters. Therefore, in this paper, a method to combine the rigorous coupled wave method suitable to the periodic structure in the vector analysis method and the genetic optimization algorithm is introduced to establish the evaluation function of the wideband filter, and the best grating structural parameters of the binary simple periodic sub-wavelength structure are obtained to satisfy the broadband filtering effect.

The goal of this paper is to design a terahertz wideband filter with a center frequency of around $4.07 \mathrm{THz}$, with a 3-dB bandwidth above $2.2 \mathrm{THz}$. The average transmission efficiency error and flatness error of the main and side lobes were selected as the factors of fitness function. The waveform was divided into three parts: the left-side lobe, the main lobe, and the right-side lobe. The definition of the transmission efficiency $T r$ is the ratio of the intensity of the outgoing wave light field to the intensity of the incident wave field. The average transmission efficiency Tra is defined in Equation (1):

$$
\operatorname{Tr} a=\frac{\operatorname{Tr}_{1}+\operatorname{Tr}_{2}+\operatorname{Tr}_{3}+\ldots \ldots+\operatorname{Tr}_{n}}{n}
$$

which means discretely dividing a frequency band into $n$ discrete points, dividing the sum of the transmission efficiencies of each discrete point. The average transmission efficiency error $M_{i}$ is defined in Equation (2):

$$
M_{i}=\left|T_{i}-\operatorname{Tr} a\right|, i=1,2,3,4,
$$

which means the absolute value of the difference between the ideal transmission efficiency Ti and the average transmission efficiency Tra, where $T i$ is 1 for the main lobe and 0 for the side lobes. The average transmission efficiency errors of the three segments are defined as $M, M_{2}$, and $M_{3}$, respectively, and the transmission efficiency error at the center frequency is $M_{4}$. The flatness error $N$ is defined as Equation (3):

$$
N_{i}=\frac{T r_{\max }-T r_{\min }}{T r_{\max }+T r_{\min }},(i=1,2,3),
$$

where each frequency domain, the difference between the highest transmission efficiency and the lowest transmission efficiency is divided by the sum of the highest transmission efficiency and the lowest transmission efficiency. If the value of $N_{i}$ is smaller, it indicates that the waveform is flatter. The flatness errors of each segment are denoted as $N_{1}, N_{2}$, and $N_{3}$, respectively.

According to the design goal requirements, in order to ensure the filter characteristics to approach the idea curve as shown in Figure 2, the following objective functions are established:

$$
G(X)=\sum_{i=1}^{3}\left(P_{i} M_{i}+Z_{i} N_{i}\right)+P_{4} M_{4}
$$

where $X$ is a collection of structural parameters, including $(f, d, h)$. The first six terms consider the transmission efficiency and flatness of each frequency segment. The seventh term considers the transmission efficiency of the center operating frequency. $\mathrm{P} i$ and $\mathrm{Zi}$ are weighting factors, which take values in the range [0,1] with their sum equal to 1 , which can be adjusted according to different design requirements. The essence of the design is to find a set of binary simple periodic sub-wavelength structure parameters $(f, d, h)$ to make sure the function $G$ is the smallest.

The frequency range of an incident terahertz wave is from 1.66 to $8.40 \mathrm{THz}$, and a wave of TE polarization is perpendicularly incident-an ordinary terahertz source is polarized light, 
and sub-wavelength devices are related to the polarization of incident light. In this paper, for simplicity, we take TE incident light as an example, where the incident medium is air of refractive index $n_{0}=1$, and the grating dielectric material is silicon of refractive index $n r=3.42$, which is almost transparent. Combined with genetic algorithm optimization, the optimal filtering effect of the filter is shown in Figure 2.

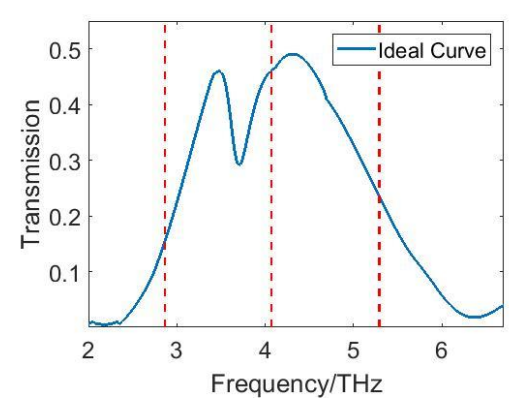

Figure 2. Optimal filtering effect.

Through actual optimization, we set the stall generation, coding method, crossover function, scaling function, generation, hybrid function, etc. Before the optimization started, the fitness of the corresponding target environment of each individual in the initial population was obtained. By finding the individual with the highest fitness in the population as the optimal individual in the population, the optimal design result searched by the genetic optimization algorithm was obtained. At the same time, the genetic optimization algorithm was very simple, adaptable, robust, and could process optimization results in parallel. Additionally, the convergence speed was quite fast. Consequently, we obtained the structural parameters of the filter $d=129.817 \mu \mathrm{m}$, the duty ratio $f=0.432$, and the groove depth $h=30.379 \mu \mathrm{m}$, with the highest transmission efficiency in the main lobe reaching close to $50 \%$. Compared with DCF, the highest transmittance was increased to $47 \%$ from $37 \%$, and 3-dB bandwidth was increased to $2.33 \mathrm{THz}$ from $2.20 \mathrm{THz}$, indicating that this work uses a simple structure to achieve a high-transmission efficiency terahertz wideband filter that breaks through the design limitations of the abovementioned terahertz filtering.

\section{Redundancy Analysis}

Due to manufacturing errors, the structural parameters of the terahertz wideband filter will affect the performance of the filter (i.e., 3-dB bandwidth and average diffraction efficiency in the filter band). It is necessary to study the redundancy of each structural parameter to ensure device performance.

The effect of the period on the transmission efficiency at each filter frequency point is shown in Figure 3. When the duty ratio and the groove depth were fixed to the design value, we swept from 100 to $180 \mu \mathrm{m}$, containing the design value of $129.817 \mu \mathrm{m}$. Figures $3 \mathrm{a}$ and $3 \mathrm{~b}$ show the effect diagram and the filter transmission spectrum. Figures $3 \mathrm{c}$ and $3 \mathrm{~d}$ are graphs of the $3-\mathrm{dB}$ bandwidth and the average transmission efficiency of each sampling point in the main lobe as a function of period. 


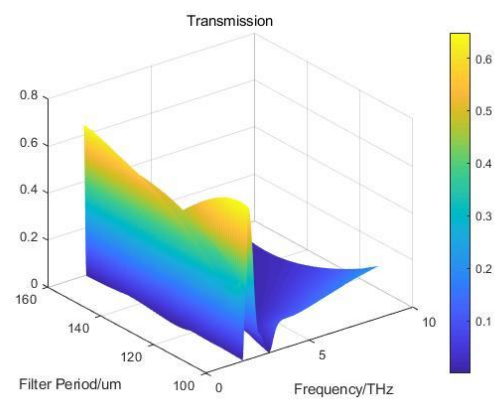

(a)

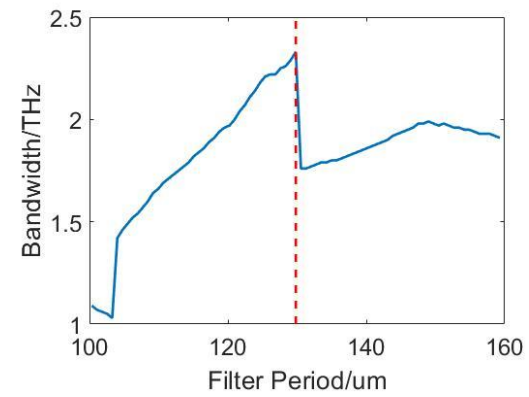

(c)

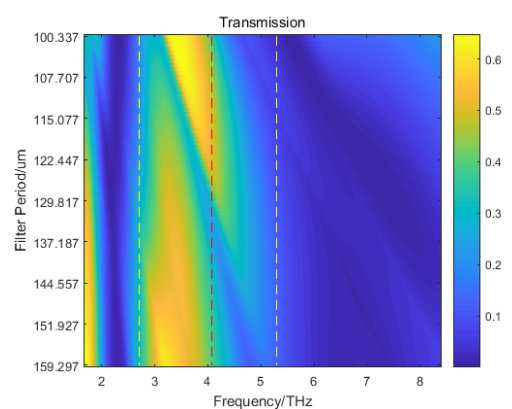

(b)

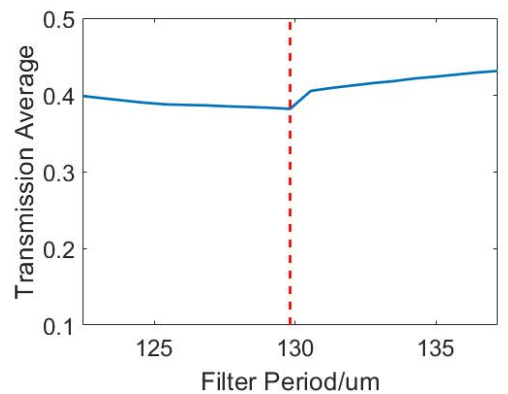

(d)

Figure 3. Effect of periodic variation on filter performance: (a) and (b) show the effect diagram and the filter transmission spectrum; (c) and (d) are graphs of the 3-dB bandwidth and the average transmission efficiency of each sampling point in the main lobe as a function of period.

As shown in Figure 3c, when the grating period was more than $129.817 \mu \mathrm{m}$, the 3-dB bandwidth of the filter dropped rapidly below $1.8 \mathrm{THz}$. When the grating period was $5.5 \mu \mathrm{m}$ less than $129.817 \mu \mathrm{m}$, the 3-dB bandwidth of the filter dropped to $2 \mathrm{THz}$ or less. It can be seen from Figure $3 \mathrm{~d}$ that there was a slight increase in the grating period of $131 \mu \mathrm{m}$, and the average transmission efficiency in the main lobe was a relatively flat straight line. Therefore, it can be considered that the grating period had a significant influence on the 3-dB bandwidth of the filter, but the average transmission efficiency within the inside lobe hardly changed around the value of $40 \%$.

When the period and groove depth were fixed to the design value and the duty ratio changed around the design value of 0.432 , the effect on the transmission efficiency at each filter frequency point was as shown in Figure 4.

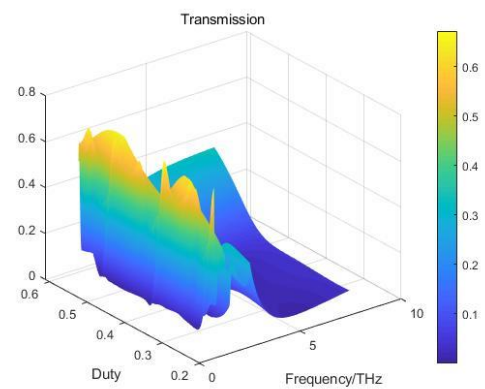

(a)

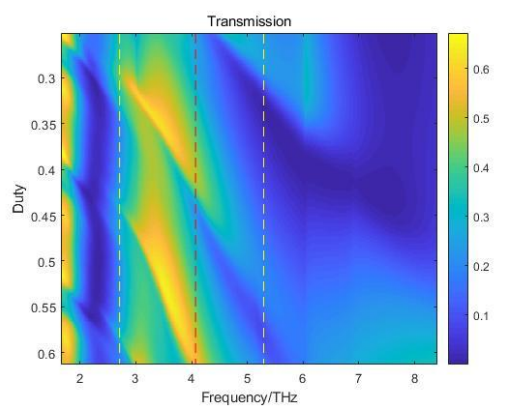

(b)

Figure 4. Cont. 


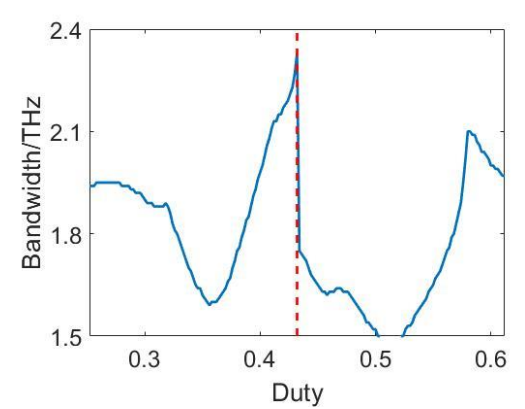

(c)

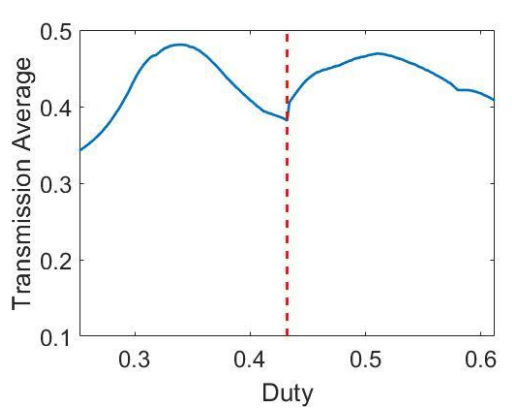

(d)

Figure 4. Effect of duty ratio variation on filter performance: (a) and (b) show the effect diagram and the filter transmission spectrum; (c) and (d) are graphs of the 3-dB bandwidth and the average transmission efficiency with different duty ratios.

As shown in Figure 4c, when the duty ratio was less than 0.041, the 3-dB bandwidth of the filter dropped to less than $2 \mathrm{THz}$. Meanwhile, when the duty ratio was shifted by 0.041 or more, and the 3-dB bandwidth of the filter fell sharply below $1.8 \mathrm{THz}$. The graph shows a relatively gentle curve in Figure $4 \mathrm{~d}$ when the average transmission efficiency in the main lobe was substantially above $40 \%$. Therefore, the duty ratio shift had a significant effect on the 3-dB bandwidth of the filter, but it did not affect the average transmission efficiency in the main lobe of the filter. When the duty ratio was less than 0.041 , the $3-\mathrm{dB}$ bandwidth was above $2 \mathrm{THz}$ - which is $15 \%$ less than that of the designed value of the ideal filter-and the average transmission efficiency in the filter main lobe was kept at about $40 \%$.

When the period and duty ratio were fixed to the design value $30.379 \mu \mathrm{m}$ and the groove depth changed, the effect on the transmission efficiency at each filter frequency point was as shown in Figure 5.

When the grating period was kept at $129.817 \mu \mathrm{m}$ and grating duty ratio at 0.432 , the grating groove depth was shifted around the ideal design value of $30.379 \mu \mathrm{m}$, and the corresponding influence on the transmission efficiency at each filtering frequency point is shown in Figure 5.

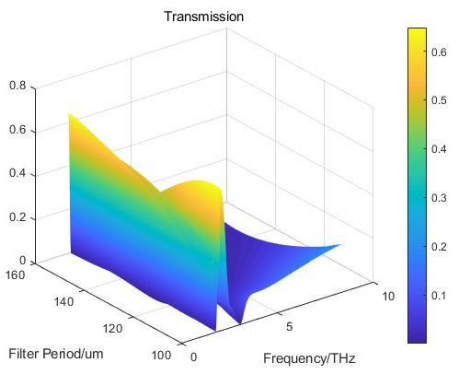

(a)

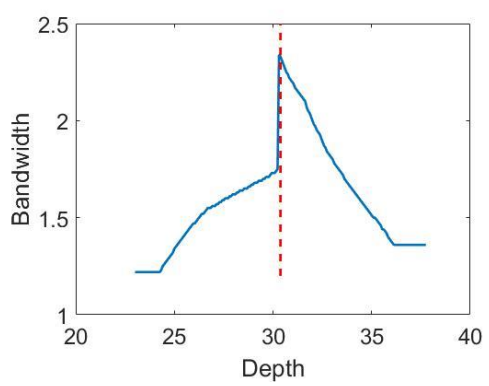

(c)

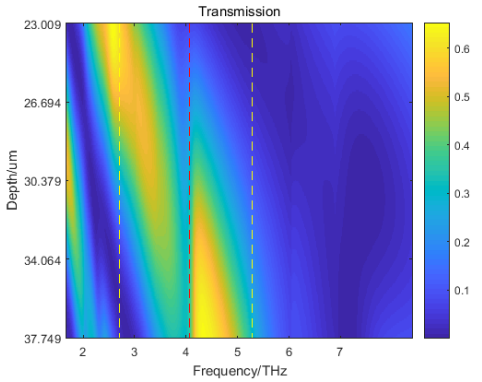

(b)

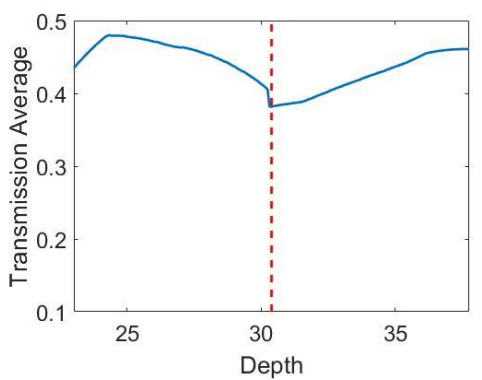

(d)

Figure 5. Effect of groove depth variation on filter performance: (a) and (b) show the effect diagram and the filter transmission spectrum; (c) and (d) are graphs of the 3-dB bandwidth and the average transmission efficiency with different groove depths. 
As shown in Figure $5 c$, when the groove depth was less than $30.379 \mu \mathrm{m}$, the 3 -dB bandwidth of the filter dropped sharply to less than $1.7 \mathrm{THz}$, while when the groove depth was shifted $1.4 \mu \mathrm{m}$ more than the design value, the 3- $\mathrm{dB}$ bandwidth of the filter dropped under $2 \mathrm{THz}$. It can be seen from Figure $5 \mathrm{~d}$ that the average transmission efficiency in the main lobe remained at nearly $40 \%$, and the graph shows a relatively gentle curve. One can conclude that groove depth shift had a significant effect on the 3-dB bandwidth of the filter, but it did not affect the average transmission efficiency in the main lobe of the filter significantly. The slot depth was in the range of $1.4 \mu \mathrm{m}$, the 3-dB bandwidth was above $2 \mathrm{THz}$, the error from the ideal design value of 3-dB bandwidth $2.33 \mathrm{THz}$ was less than 15\%, and the average transmission efficiency in the filter main lobe was kept at around $40 \%$.

In order to discuss the influence of a single parameter on the filtering effect, the above control variables method was performed by fixing other parameters. However, all structural parameters in the actual production will deviate from the expected ideal design value. Therefore, this paper conducts a comprehensive error analysis on the design of the terahertz wideband filter so that the structural parameters $(f, d, h)$ can be chosen in certain ranges, allowing the structural parameters to make the deviance as large as possible in order to reduce difficulties in the actual production. After calculation, the variation ranges of each structural parameter for obtaining the best filtering performance were: grating period from 125.4 to $130.5 \mu \mathrm{m}$ (relative offset $127.9 \pm 2.5 \mu \mathrm{m}$ ); grating duty ratio from 0.412 to 0.417 (relative offset $0.414 \pm 0.002$ ); minimum line width redundancy $\pm 0.4 \mu \mathrm{m}$; and grating groove depth from $28.9 \mu \mathrm{m}$ to $30.3 \mu \mathrm{m}$ (relative offset was $29.6 \pm 0.7 \mu \mathrm{m}$ ). Note that the structural parameters satisfy current fabrication technology. The terahertz wideband filter had a 3-dB bandwidth above $2 \mathrm{THz}$ and an average transmission efficiency of around $40 \%$ in the main lobe. The best fabrication structure parameters were selected as follows: grating period of $127.9 \mu \mathrm{m}$; duty ratio of 0.414 (the minimum line width was $53 \mu \mathrm{m}$ ); and grating groove depth of $29.6 \mu \mathrm{m}$. The current mature micro-nano manufacturing technology meets the manufacturing requirements of the structure.

\section{Production and Performance Testing}

In this paper, a sample was fabricated with inductively coupled plasma reactive ion etching technology. The three-dimensional and top views of the fabricated terahertz broadband filter are shown in Figure 6.

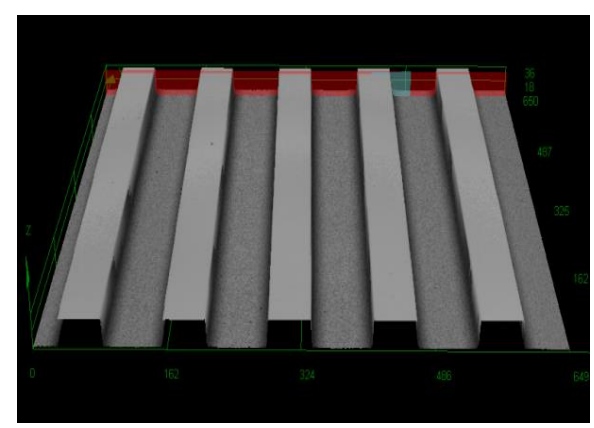

(a)

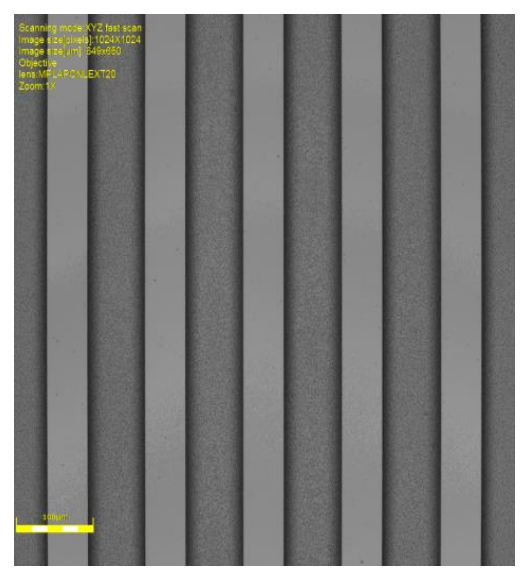

(b)

Figure 6. (a) SEM 3D and (b) SEM top view of the terahertz wideband filter.

According to the SEM measurement, the groove depth of the actual fabricated terahertz broadband filter was about $29.81 \mu \mathrm{m}$, which is close to the design value of $29.60 \mu \mathrm{m}$ and is within the error tolerance. The period of the fabricated terahertz wideband filter was $127.19(53.00+74.19) \mu \mathrm{m}$, which is close to the design value of $127.90 \mu \mathrm{m}$ and is within the error tolerance. The duty ratio of the fabricated 
terahertz wideband filter was $0.417(53.00 / 127.19)$ and the design value is 0.414 , which is within the error tolerance.

The sanple was tested in Shanghai THz device testing company (Shanghai, China), and Advantest TAS7500 series terahertz band ultra-wideband spectral analysis system (Advantest, Shanghai, China) was used to test the filter performance. After the analysis unit received the analysis signal and transmitted it to the PC, the experimental test data were obtained. The test results of the sample were basically the same as the curve of the simulation results. As shown in Figure 7, the corresponding frequency point at the highest transmission efficiency peak was close to $4.07 \mathrm{THz}$, and there was a significant transmission efficiency through near $6 \mathrm{THz}$. However, the spectral line of the test results exhibited a multi-spike shape because the terahertz wave was generated by the ultrafast laser-pulsed nonlinear crystal, which resulted in the selection of the terahertz signal by the cavity.

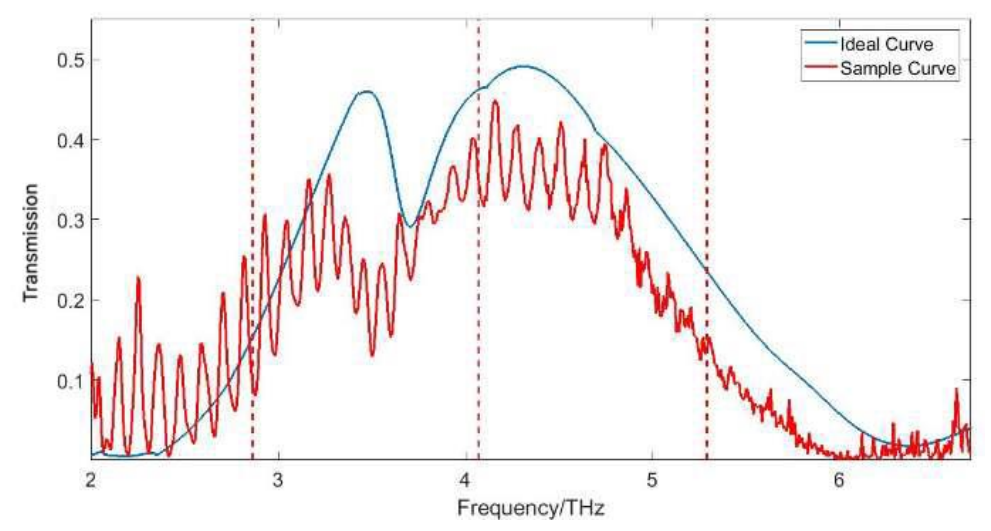

Figure 7. Comparison of sample simulation filter curve and actual measurement filter curve.

The peak structure of the above test data interfered with the calculation of 3-dB bandwidth to a certain extent, and also affected the quantitative comparison and analysis of experimental data and simulation data. In order to better perform data analysis, the measured results were polynomial-fitted, and the frequencies of the measured data were subdivided into many different frequency bands, where each frequency point corresponded to a transmission efficiency of the point, and the peak point of each pulse in each frequency band was selected and relabeled. Finally, the fitted envelope was compared with the pre-fit experimental value and the sample simulation optimization result, as shown in Figures 8 and 9:

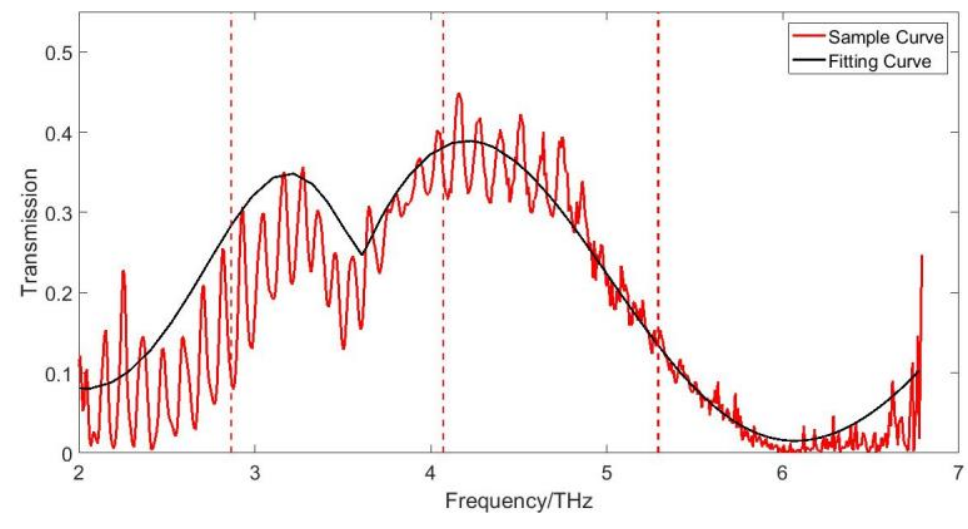

Figure 8. Comparison of actual measurement curve and fitted curve. 


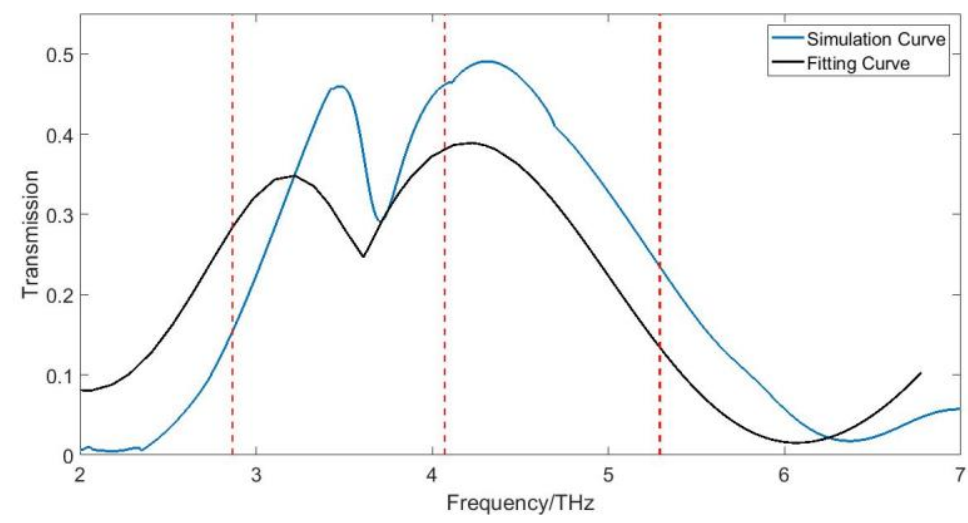

Figure 9. Comparison of sample simulation curve and fitted curve.

Figure 9 shows that the fitting curve agreed well with the sample test value, and the black fitting curve was completely in the middle of the red sample test data peak structure. In the measurement, the 3-dB bandwidth of the fitting curve was $2.18 \mathrm{THz}$, and the highest transmission efficiency was $38.1 \%$ in the main lobe. In the simulation, the $3-\mathrm{dB}$ bandwidth was $2.19 \mathrm{THz}$, the highest transmission efficiency was $48.8 \%$, as shown in Table 1 . The reason for the declining transmission efficiency in the measurement consists of three main parts: firstly, the absorption of the material itself caused the loss of the transmitted wave energy; secondly, for the incident beam, the incident condition was approximately of normal incidence; finally, the collimation beam expansion process was not performed, and there was a space transmission loss of the terahertz beam in the atmosphere.

Table 1. Comparison of actual production and theoretical design of the filtering effect of the terahertz wideband filter.

\begin{tabular}{ccc}
\hline Value & 3-dB Bandwidth & Maximum Transmission Efficiency \\
\hline Designed & $2.21 \mathrm{THz}$ & $49.1 \%$ \\
\hline Sample (simulated) & $2.19 \mathrm{THz}$ & $48.9 \%$ \\
\hline Sample (tested) & $2.18 \mathrm{THz}$ & $38.1 \%$ \\
\hline
\end{tabular}

\section{Conclusions}

In this paper, a rigorous coupled wave method and a genetic algorithm were used to propose a sub-wavelength binary simple periodic structure broadband filter design method which effectively realizes the filtering effect of broadband and high diffraction efficiency.

A center frequency of $4.07 \mathrm{THz}$ was designed and filtered. A terahertz wideband filter with a frequency range of $2.94-5.27 \mathrm{THz}$ was used to fabricate samples and tested using a binary optical process. The experimental results were consistent with the design results. As shown in Table 2, the highest transmission efficiency in the main lobe was close to $50 \%$, the highest transmittance of the reference double cone filter was increased by more than $10 \%$, the 3-dB bandwidth was $2.33 \mathrm{THz}$, and the width was $0.13 \mathrm{THz}$. The influence of the variation of the grating structure parameters on the filtering effect of the filter was studied. The variation of the structural parameters had a great influence on the bandwidth. It was especially sensitive to the variation of the groove depth. Groove depth error control should be strengthened during the production. The fabrication error of the terahertz broadband filter structure parameters was analyzed, and the most suitable fabrication parameters are given: a grating period of $127.9 \mu \mathrm{m}$; a duty ratio of 0.414 ; and a grating groove depth of 29.60 $\mu \mathrm{m}$. The allowable structural parameter redundancy ranges include: a grating period of $127.9 \mu \mathrm{m}$ $( \pm 2.5 \mu \mathrm{m})$; a grating duty ratio of $0.414( \pm 0.002$, minimum line width $53.0 \mu \mathrm{m}$, redundancy \pm 0.4 $\mu \mathrm{m})$; and a grating groove depth of $29.6 \pm 0.7 \mu \mathrm{m}$. The current production technology can fully realize the structure. The device was fabricated and tested with a 3-dB bandwidth of $2.18 \mathrm{THz}$, which 
is consistent with the design results, proving the correctness and feasibility of the design method. Additionally, there was a maximum transmission efficiency of $38.1 \%$ in the main lobe, which is less than the simulation and the reasons for this are discussed.

Table 2. Comparison of our work and double-cone filter (DCF) terahertz wideband filter.

\begin{tabular}{ccc}
\hline Wideband Filter & 3-dB Bandwidth & Maximum Transmittance in the Main Lobe \\
\hline DCF & $2.20 \mathrm{THz}$ & $37.0 \%$ \\
\hline Our work & $2.33 \mathrm{THz}$ & $50.9 \%$ \\
\hline
\end{tabular}

In this paper, a high-performance terahertz wideband filter was realized that overcomes the limitations of the current terahertz filters with a simple structure, a lower cost, and easier production. At the same time, tolerance analysis and manufacturing error analysis of the structural parameters were carried out. The actual sample preparation and performance test showed that the test results were consistent with the theoretical design, and can be applied in the fields of terahertz communication, imaging, and other fields.

Author Contributions: Conceptualization, P.Y., L.L. and X.P.; Data curation, H.H.F., Z.Y. and X.Y.F.; Formal analysis, Y.T.; Funding acquisition, H.H.F.; Investigation, P.Y.; Methodology, P.Y.; Project administration, L.L. and X.P.; Resources, Y.T.; Software, Z.Y. and X.Y.F.

Funding: This work has been supported by grants from the National Natural Science Foundation of China (No. 61275167), the Basic Research Project of Shenzhen (JCYJ20130329103020637, JCYJ20140418095735591, JCYJ20160603172549843, JCY20170817102315892 and JCYJ2017081701827765).

Conflicts of Interest: The authors declare no conflict of interest.

\section{References}

1. Biber, S.; Hofmann, A.; Schulz, R.; Collischon, M.; Weinzierl, J.; Schmidt, L.-P. Design and measurement of a bandpass filter at $300 \mathrm{GHz}$ based on a highly efficient binary grating. IEEE Trans. Microw. Theory Tech. 2004, 52, 2183-2189. [CrossRef]

2. Lee, J.; Seo, M.; Park, D.; Kim, D.; Jeoung, S.; Lienau, Ch.; Park, Q.H.; Planken, P. Shape resonance omni-directional terahertz filters with near-unity transmittance. Opt. Express 2006, 14, 1253-1259. [CrossRef] [PubMed]

3. Libon, I.H.; Baumgärtner, S.; Hempel, M.; Hecker, N.E.; Feldmann, J. An optically controllable terahertz filter. Appl. Phys. Lett. 2000, 76, 2821-2823. [CrossRef]

4. Han, N.R.; Chen, Z.C.; Lim, C.S.; Ng, B.; Hong, M.H. Broadband multi-layer terahertz metamaterials fabrication and characterization on flexible substrates. Opt. Express 2011, 19, 6990-6998. [CrossRef] [PubMed]

5. Park, H.R.; Park, Y.M.; Kim, H.S.; Kyoung, J.S.; Seo, M.A.; Park, D.J.; Ahn, Y.H.; Ahn, K.J.; Kim, D.S. Terahertz Nano resonators: Giant Field Enhancement and Ultrabroad Band Performance. Appl. Phys. Lett. 2010, 96, 1-3. [CrossRef]

6. Zhou, X.; Yin, X.; Zhang, T.; Chen, L.; Li, X. Ultrabroad terahertz bandpass filter by hyperbolic metamaterial waveguide. Opt. Express 2015, 23, 11657-11664. [CrossRef] [PubMed]

7. Zhu, M.; Lee, C. A Design of Terahertz Broadband Filters and its Effect in Eliminating Asymmetric Characteristics in Device Structures. J. Light. Technol. 2015, 33, 3280-3285. [CrossRef]

8. Li, Z.; Ding, Y.J. Broadband stopband filter for terahertz wave based on multi-layer metamaterial microstructure. In Proceedings of the 2012 Conference on Lasers and Electro-Optics (CLEO), San Jose, CA, USA, 6-11 May 2012.

9. Villa, C.; Hayduk, M.; Donkor, E. Terahertz Optical Frequency Comb Generation by Spectral Filtering of Broadband Spontaneous Amplified Emissions from a Semiconductor Optical Amplifier. J. Light. Technol. 2009, 27, 5437-5441. [CrossRef]

10. Xu, P.; Yuan, X.; Huang, H.; Yang, T. Research on Design of Tri-color Shift Device. Nanoscale Res. Lett. 2016, 11, 485. [CrossRef] [PubMed] 
11. Huang, H.; Ruan, S.; Yang, T.; Xu, P. Novel even beam splitters based on subwavelength binary simple periodic rectangular structure. Nano-Micro Lett. 2015, 7, 177-182. [CrossRef]

12. Xu, P.; Hong, C.; Cheng, G.; Liang, Z.; Sun, Z. Planar optical correlators integrated with binary optical lens. Opt. Express 2015, 23, 6773-6779. [CrossRef] [PubMed]

13. Xu, P.; Huang, Y.; Su, Z.; Zhang, X.; Luo, T.; Peng, W. Research of micro-prism distribution on the bottom surface of the small-size integrated light guide plate. Opt. Express 2015, 23, 4887-4896. [CrossRef] [PubMed]

14. Xu, P.; Huang, Y.; Zhang, X.; Huang, J.; Li, B.; Ye, E.; Duan, S.; Su, Z. Integrated micro-optical light guide plate. Opt. Express 2013, 21, 20159-20170. [CrossRef] [PubMed]

15. Xu, P.; Huang, H.; Wang, K.; Ruan, S.; Yang, J.; Wan, L.; Chen, X.; Liu, J. Realization of optical perfect shuffle with microoptical array element. Opt. Express 2007, 15, 809-816. [CrossRef] [PubMed]

16. Xu, P.; Huang, Y.; Su, Z.; Zhang, X. Algorithm research on microstructure distribution on the bottom surface of an integrated micro-optical light guide plate. Appl. Opt. 2014, 53, 1322-1327. [CrossRef] [PubMed]

17. Xu, P.; Li, X.; Yang, T.; Sun, Z.; Huang, H.; Yu, J.; Cheng, G. Research on distortion invariant recognition based on lensless coaxial integrated microoptic correlator. J. Opt. 2018, 20, 085801. [CrossRef]

18. Xu, P.; Yang, T.; Huang, H.; Li, X.; Xiao, Y.; Pan, Y.; Yang, W. Research on distortion invariant recognition of planar integrated optical correlator. IEEE Photonics J. 2018, 10, 7800410. [CrossRef]

19. Xu, P.; Yang, T.; Liang, K.; Li, X.; Long, S.; Huang, H. A Novel Method to Realize Optical Correlation Recognition Based on Neural Network. IEEE Photonics J. 2018, 10, 7801210. [CrossRef]

(C) 2019 by the authors. Licensee MDPI, Basel, Switzerland. This article is an open access article distributed under the terms and conditions of the Creative Commons Attribution (CC BY) license (http://creativecommons.org/licenses/by/4.0/). 